\title{
WAVELET-BASED IDENTIFICATION AND CLASSIFICATION OF LOCAL SYMMETRIES IN MICROSCOPY IMAGES
}

\author{
Zsuzsanna Püspöki, Student Member, IEEE, and Michael Unser, Fellow, IEEE
}

\author{
Biomedical Imaging Group, École polytechnique fédérale de Lausanne (EPFL), Switzerland
}

\begin{abstract}
We present a method for the identification and classification of local symmetries in biological images. We aim at obtaining a precise estimate of symmetric junctions in a scale and rotation invariant way. The proposed method is template-free, which allows the test of any combination of arbitrary symmetry orders in an effective way.

Our measure of local symmetry is derived from a circular harmonic wavelet analysis. The basis functions exhibit different symmetry orders. We use this measure to formulate a classifier to label the different junctions into one of several symmetry classes.

We present experimental results, and validate our method using both on synthetic images and biological micrographs.
\end{abstract}

\section{INTRODUCTION}

Local symmetries and symmetric junctions are common in nature. For instance, bilateral and three-fold symmetric objects can be found in various biological samples: two-fold symmetries (ridges) are present in filaments, fibers and membranes; hexagonal patterns appear in endothelial cells (e.g. in the eyes), in the leaf or stem cross-section of plants (e.g. Convallaria), etc. The detection of these symmetries and junctions is fundamental to analysis of the corresponding microscopic images. As an example, experiments in stem cell research require the accurate detection of cell structures (like tight junctions), which exhibit polygonal shapes [1].

The difficulty of the problem is twofold. First, patterns in natural images are frequently subjected to unknown and variable geometric transformations such as rotation, translation, and scaling. Second, biological micrograps are affected by various types of distortion, such as measurement noise (Poisson, etc.) and local variations in intensity.

Within the framework of circular harmonic wavelet frames, we propose a method to overcome these problems in order to the detect and classify local symmetries in an efficient way.

Broadly, one can separate approaches targeting junction detection into three categories: methods based on the detection and analysis of edges and gradients [2,3]; feature analysis with structure tensors $[4,5]$; or matching parametric junction templates [6$11]$.

Detectors based on gradient or the structure tensor are commonly used in computer vision. They are mostly adapted to differentiate between specific types of keypoints (e.g. junction, or edge). Detectors in the last category are usually template-based,

This work was partially supported by the ERC (ERC-2010-AdG 267439FUN-SP), the Hasler Foundation and the Indo-Swiss Joint Research Program and, with the exception of steerable filters, they involve discretized rotation angles for template-matching.

In this paper, we propose a template-free method for the identification and classification of local symmetry. The backbone of our algorithm is the circular harmonic wavelet transform, which distributes the energy of the image among a set of harmonics corresponding to different symmetry orders. Relying on this energy distribution, we propose a measure of symmetry and a framework for classification of symmetry order based on assigning energies to different symmetry states and identifying the minimum energy state.

Our method differs from the existing approaches in several ways. First of all, our framework is template-free, thus there is no need for template-matching, which is computationally heavy and less accurate. Second, our framework has a multiresolution structure, which provides detections across scales. Finally, our system provides a measure of symmetry at each location in the image and for arbitrary symmetry orders in a fast and robust way. It is insensitive to local variations in image intensity.

\section{GENERAL FRAMEWORK}

To introduce the notations and set the basis of our algorithm, we give a brief introduction to circular harmonic wavelet frames, and we refer to [12] for more details. The symmetry measure (score) and the classification problem are discussed next.

In this paper we use $\boldsymbol{x} \in \mathbb{R}^{2}$ and $\boldsymbol{\omega} \in \mathbb{R}^{2}$ for the Cartesian, and $(r \in \mathbb{R}, \theta \in[-\pi, \pi)$ ) and $(\rho \in \mathbb{R}, \phi \in[-\pi, \pi)$ ) for the polar coordinates in spatial and Fourier domains, respectively. We denote the Fourier transform of a function $f$ by $\hat{f}$.

\subsection{Circular Harmonic Wavelet Frames}

To construct a circular harmonic tight frame we start from a bandlimited isotropic mother wavelet in $\mathrm{L}_{2}\left(\mathbb{R}^{2}\right)$, denoted by $\psi$, whose shifts and dilations form a wavelet frame. This isotropic wavelet at scale $i$ and grid point (location) $\boldsymbol{x}_{0}$ (in 2D), has the form

$$
\psi_{i}\left(\boldsymbol{x}-\boldsymbol{x}_{0}\right)=\frac{1}{2^{i}} \psi\left(\frac{\boldsymbol{x}-\boldsymbol{x}_{0}}{2^{i}}\right)
$$

In this work, we use Simoncelli's isotropic mother wavelet [13], defined by its Fourier transform:

$$
\hat{\psi}(\rho)= \begin{cases}\cos \left(\frac{\pi}{2} \log _{2}\left(\frac{2 \rho}{\pi}\right)\right), & \frac{\pi}{4}<\rho \leq \pi \\ 0, & \text { otherwise }\end{cases}
$$

The circular harmonic wavelet frame is obtained from this primal isotropic wavelet by the application of the multi-order complex Riesz transform. 
The $n$ th-order complex Riesz transform, $\mathscr{R}^{n}$, is the $n$-fold iterate of the complex Riesz transform, $\mathscr{R}$, defined in the Fourier domain as

$$
\mathscr{R}^{n} f(\boldsymbol{x}) \leftrightarrow \mathrm{e}^{\mathrm{j} n \phi} \hat{f}_{\text {pol }}(\rho, \phi) .
$$

It is a unitary, scale-invariant transform, whose application on $\psi_{i}\left(\cdot-\boldsymbol{x}_{0}\right)$ preserves its tight frame property. It means that, by choosing a distinct set of harmonics ( $N$ distinct values for the integer $n$, constituting the set $S$ of harmonics) we can form a tight frame consisting of $n$ channels, referred to as circular harmonic wavelets.

Proposition 1 The wavelet channels corresponding to harmonics $n_{1}$ and $n_{2}$ with $n_{1} \neq n_{2}$ are orthogonal to each other.

Proof.

To prove that the generated wavelet channels at a given scale $i$ and position $\boldsymbol{x}_{0}$ are orthogonal, note that

$$
\begin{aligned}
& \left\langle\psi_{i}^{\left(n_{1}\right)}\left(\cdot-\boldsymbol{x}_{0}\right), \psi_{i}^{\left(n_{2}\right)}\left(\cdot-\boldsymbol{x}_{0}\right)\right\rangle \\
& =\frac{2^{2 i}}{(2 \pi)^{2}} \int \hat{\psi}^{\left(n_{1}\right)}\left(2^{i} \boldsymbol{\omega}\right) \overline{\hat{\psi}^{\left(n_{2}\right)}\left(2^{i} \boldsymbol{\omega}\right)} \mathrm{d} \boldsymbol{} \\
& =\frac{2^{2 i}}{(2 \pi)^{2}} \int_{0}^{\infty} \int_{-\pi}^{\pi}\left|\hat{h}\left(2^{i} \rho\right)\right|^{2} \mathrm{e}^{\mathrm{j} n_{1} \phi} \mathrm{e}^{-\mathrm{j} n_{2} \phi} \rho \mathrm{d} \rho \mathrm{d} \phi \\
& =\frac{1}{(2 \pi)^{2}} \int_{0}^{\infty} \int_{-\pi}^{\pi}|\hat{h}(\rho)|^{2} \mathrm{e}^{\mathrm{j} n_{1} \phi} \mathrm{e}^{-\mathrm{j} n_{2} \phi} \rho \mathrm{d} \rho \mathrm{d} \phi \\
& =\frac{1}{(2 \pi)^{2}} \int_{0}^{\infty}|\hat{h}(\rho)|^{2} \rho \mathrm{d} \rho \int_{-\pi}^{\pi} \mathrm{e}^{\mathrm{j}\left(n_{1}-n_{2}\right) \phi} \mathrm{d} \phi \\
& =C \int_{-\pi}^{\pi} \mathrm{e}^{\mathrm{j}\left(n_{1}-n_{2}\right) \phi} \mathrm{d} \phi,
\end{aligned}
$$

where we took advantage of the Parseval identity, the isotropy of the primal radial wavelet, and a change of variables. The last integral is 1 if $n_{1}=n_{2}$, and vanishes otherwise. This means that the generated wavelet channels at a given scale $i$ and position $x_{0}$ are orthogonal.

The orthogonality is valid across wavelet channels at any given scale and location.

As a result of the transformation and the orthogonalitiy of the harmonic channels, the local energy of the image is preserved and distributed among a set of harmonics, corresponding to the angular Fourier factors $\mathrm{e}^{\mathrm{j} n \phi}$. In the following subsection we examine this distribution and use it to define a measure of local $M$-fold symmetry.

\subsection{Local Wavelet Energies}

Let $q_{n, i}$ denote the $n$th channel wavelet coefficient at scale $i$ and location of interest $x_{0}$ :

$$
q_{n, i}=\left\langle\psi_{i}^{(n)}\left(\cdot-x_{0}\right), f\right\rangle .
$$

To establish a correspondence with the symmetry order, we define the following energy term for $n \in S$ :

$$
E_{n, i}=\frac{\left|q_{n, i}\right|^{2}}{\sum_{m \in S}\left|q_{m, i}\right|^{2}},
$$

to which we refer to as normalized-energy wavelet coefficients. In Figure 1, we show the distribution of the normalized-energy wavelet coefficients at a fixed scale and four different locations:
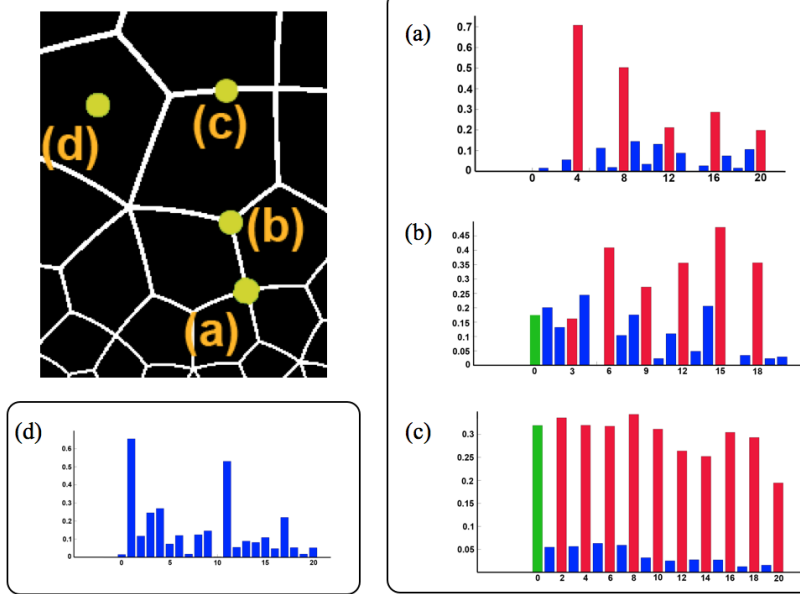

(b)

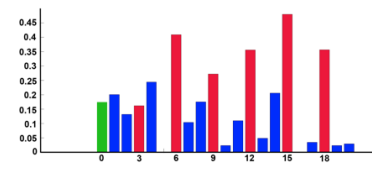

(c)

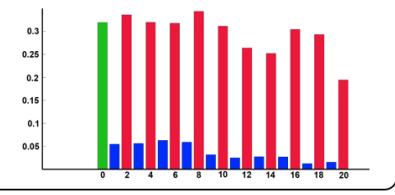

Fig. 1. The distribution of normalized-energy wavelet coefficients corresponding to 4 different locations: (a), (b), (c) and (d).

(a) four-fold symmetry (cross-intersection), (b) three-fold symmetry, (c) two-fold symmetry (ridge), (d) no symmetry. In the case of a cross-intersection, we observe that every fourth component is high compared to the rest. In case of three-fold symmetry this is valid for every third, while in case of the ridge, every second. By contrast, when we lack symmetry no such concentration is observed. Thus, empirically we can say that the "energy" of the wavelet is highly concentrated in $M$-fold periodic harmonics in case of $M$-fold symmetry, while there is no repetitive pattern in the absence of local symmetries. We use this information to build up a model for the quantitative analysis of the data.

\subsection{Symmetry Metrics}

Based on the above discussion we define the following quantity ( $\mathrm{F}$ score) as a measure of symmetry:

$$
F\left(\boldsymbol{x}_{0} ; M, i\right)=\frac{\frac{1}{\left|S_{M}\right|} \sum_{n \in S_{M}}\left|q_{n, i}\right|^{2}}{\frac{1}{\left|S-S_{M}\right|} \sum_{n \in S-S_{M}}\left|q_{n, i}\right|^{2}},
$$

for a fixed scale $i$ and location $x_{0} . S_{M}$ denotes the subset of $S$ consisting of harmonics that are integer multiples of $M\left(S_{M}=\{n \in S\right.$ : $M \mid n\} \subset S)$ and $\left|S_{M}\right|$ denotes the cardinality of the set $S_{M}$. Conceptually, by separating out the $M$-periodic circular harmonics, we break up the local energy of the image around a point into a complementary component with $M$-fold angular symmetries (numerator) and a component lacking such symmetries (denominator). The ratio of these energies is used to determine if there is a significantly higher concentration of energy in the first component.

The stronger the local $M$-fold symmetry, the larger is the numerator of $F\left(x_{0} ; M, i\right)$ compared to its denominator. Thus, a higher F score indicates a stronger $M$-fold symmetry at location $\boldsymbol{x}_{0}$ and scale $i$. Moreover, this quantity is invariant to changes of intensity.

To obtain multiscale detection, we define a (dyadically) scaleinvariant measure by taking the maximum over all scales:

$$
F\left(x_{0} ; M\right)=\max _{i} F\left(x_{0} ; M, i\right) .
$$




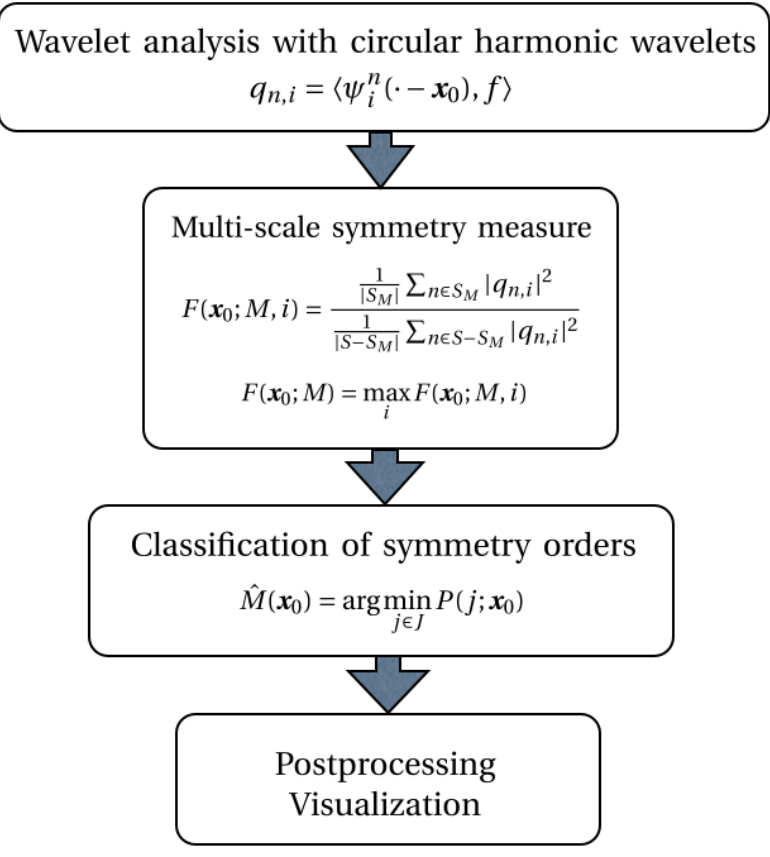

Fig. 2. The main steps of the algorithm.

\subsection{Classification of Symmetry Orders}

We use the F score, defined earlier, to classify keypoints into one of several symmetry classes. To assign a symmetry class to a given point, we take advantage of the fact that the F score indicates the strength of a local $M$-fold symmetry. Our approach is based on the comparison of $\mathrm{F}$ scores for different symmetry orders.

To classify a pixel as belonging to one of several local symmetry classes, we define a potential energy for each state (symmetry order) $j$, using the measure introduced previously:

$$
P\left(j ; x_{0}\right)=-g_{j}\left(F\left(x_{0} ; j\right)\right),
$$

where $g_{j}$ s are monotonically increasing functions. The lower potential corresponds to a stronger $j$-fold symmetry (i.e. a more stable symmetry state). Given a set $J$ of possible symmetry orders, the local symmetry order of point $\boldsymbol{x}_{0}$ is then determined by the state with the lowest potential energy:

$$
\hat{M}\left(\boldsymbol{x}_{0}\right)=\arg \min _{j \in J} P\left(j ; \boldsymbol{x}_{0}\right) .
$$

The simplest way to define the potential energy is by taking all $g_{j} \mathrm{~s}$ to be the identity function. To account for the different distribution of the scores as a function of $j$, we modify this by introducing rescaling factors $C_{j}$ depending on $j: g_{j}(u)=u / C_{j}$. For this, we assume an i.i.d. Gaussian distribution for the $q$ coefficients in (1), and set $C_{j}$ as the threshold for $F(\cdot ; j)$ to reach a fixed probability (here 0.95 ). The resulting rule is:

$$
\hat{M}\left(\boldsymbol{x}_{0}\right)=\arg \min _{j \in J}-\frac{F\left(\boldsymbol{x}_{0} ; j\right)}{C_{j}}=\arg \max _{j \in J} \frac{F\left(\boldsymbol{x}_{0} ; j\right)}{C_{j}} .
$$

\subsection{Local Symmetry Detection}

The algorithm we propose detects and classifies local symmetry centers in a multiscale and template-free way. The main steps of the algorithm are summarized in Figure 2.

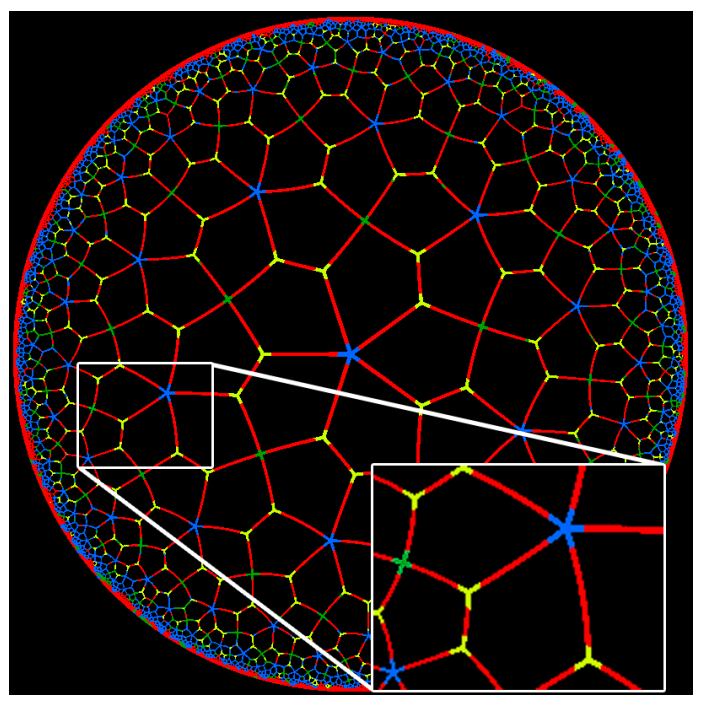

Fig. 3. Uniform 3-4-5 tiling in hyperbolic plane. Detection of twofold symmetries or ridges (red), three- (yellow), four- (green), and five-fold (blue) junctions.

First, we do a wavelet analysis with circular harmonic wavelets, which is followed by the computation of the symmetry measure in a multiscale way. Once the measures are computed for each possible symmetry order at each location, the classification of the different symmetry orders is performed. The detection process is finished after postprocessing and visualization stages.

The main postprocessing step is dedicated to improving the results by local voting in case of noise. It means that, after the classification process, the algorithm takes a majority vote in the local neighborhood of each pixel.

\section{EXPERIMENTAL RESULTS}

Our symmetry detection and classification algorithm is available as a plugin for ImageJ, a public-domain image processing platform implemented in Java. The result of the identification and classification process is a colored image, denoting the different local symmetries with different colors. The typical running time of the algorithm for two different symmetry orders taking an image of $512 \times 512$ pixels is around $10 \mathrm{sec}$ on a $2.4 \mathrm{GHz}$ Mac workstation.

To evaluate the performance of the algorithm, we used various test images. Among them we show two representative examples. The first test image that is a 3-4-5 uniform tiling of the hyperbolic plane. ${ }^{1}$ It exhibits different local symmetries in a multiscale way (Figure 3). Our goal was to identify the different local symmetries, namely: two- (ridges), three-, four- (cross intersection) and fivefold. The detections are accurate regardless of the orientation and scale of the patterns. The second test image presents a stem cross section of maize (Figure 4, upper left). As is common in plants, the cells form a type of mesh where most nodes have a fixed order (here 3). Therefore, our goal here was to distinguish between these three-fold junctions and the two-fold cell walls that connect them. Despite the non-uniform intensity and the variations in shapes and angles, as well as size and orientation, almost all three-fold junctions are detected and distinguished from the cell walls.

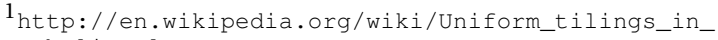
hyperbolic_plane
} 

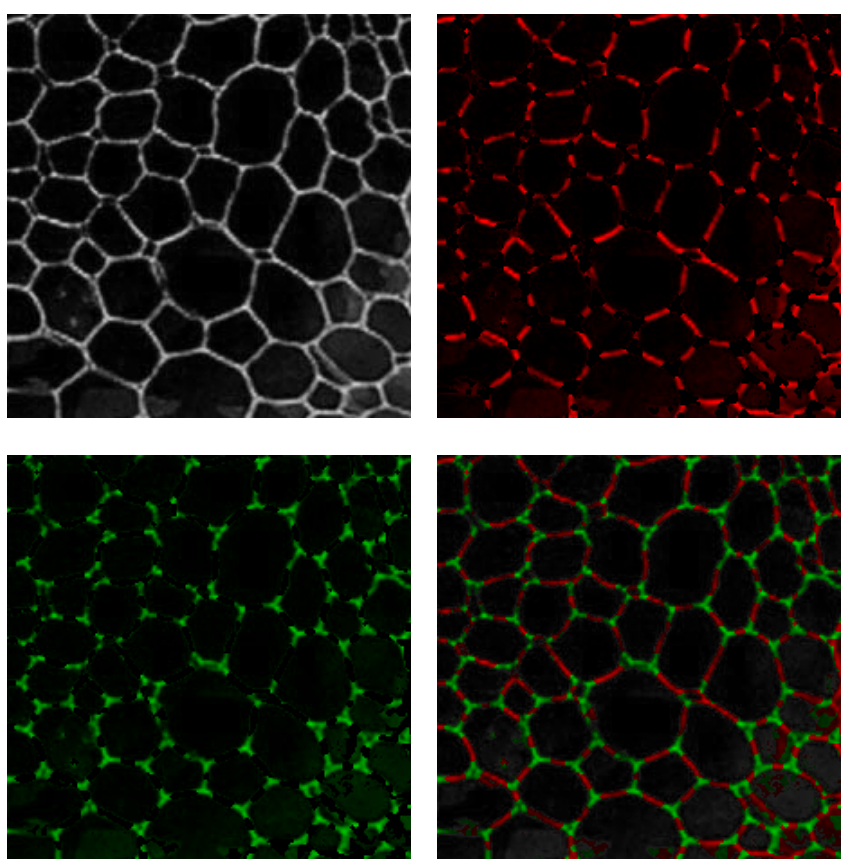

Fig. 4. Stem cross section of maize in light microscopy. Detection of two-fold symmetries or ridges (red) and three-fold junctions (green). Subfigures: original image, detected edges, detected three-fold junctions, combined colored image.

\section{CONCLUSIONS}

We presented a general wavelet-based framework for the detection and classification of local symmetry centers in images. The attractive features of the algorithm are (i) the multiscale approach and the rotation-invariance, which make it possible to obtain a precise estimate of the junctions across scales and at arbitrary orientations; (ii) robustness to noise, deformations, and local intensity variations; and (iii) speed. Also, since any combination of arbitrary symmetry orders may be tested without requiring one to design specific templates, the approach is general and easily extensible without a significant overhead for increasing the number of tested patterns.

\section{ACKNOWLEDGMENT}

This work was partially supported by the ERC (ERC-2010-AdG 267439-FUN-SP), the Hasler Foundation and the Indo-Swiss Joint Research Program.

\section{REFERENCES}

[1] F. Osakada, H. Ikeda, M. Mandai, T. Wataya, K. Watanabe, N. Yoshimura, A. Akaike, Y. Sasai, and M. Takahashi, "Toward the generation of rod and cone photoreceptors from mouse, monkey and human embryonic stem cells," Nature Biotechnology, vol. 26, no. 2, pp. 215-224, 2008.

[2] D. J. Beymer, "Finding junctions using the image gradient," A.I. Memos, M.I.T. Artifical Intelligence Laboratory, vol. AIM1266, pp. 1-46, Dec. 1991.
[3] T. A. Pham, M. Delalandre, S. Barrat, and J. Ramel, "A robust approach for local interest point detection in line-drawing images," Document Analysis Systems (DAS), 2012 10th IAPR International Workshop on, pp. 79-84, Mar. 2012.

[4] U. Koethe, "Edge and junction detection with an improved structure tensor," in Pattern Recognition, ser. Lecture Notes in Computer Science, B. Michaelis and G. Krell, Eds. Springer Berlin / Heidelberg, 2003, vol. 2781, pp. 25-32.

[5] J. Bigun, T. Bigun, and K. Nilsson, "Recognition by symmetry derivatives and the generalized structure tensor," Pattern Analysis and Machine Intelligence, IEEE Transactions on, vol. 26, no. 12, pp. 1590-1605, Dec. 2004.

[6] W. T. Freeman, "Steerable filters and local analysis of image structure," Ph.D. dissertation, Massachusetts Institute of Technology, 1992.

[7] E. Simoncelli and H. Farid, "Steerable wedge filters for local orientation analysis," Image Processing, IEEE Transactions on, vol. 5, no. 9, pp. 1377-1382, Sep. 1996.

[8] L. Parida, D. Geiger, and R. Hummel, "Junctions: detection, classification, and reconstruction," Pattern Analysis and Machine Intelligence, IEEE Transactions on, vol. 20, no. 7, pp. 687-698, Jul. 1998.

[9] F. Escolano, P. Suau, and B. Bonev, Information Theory in Computer Vision and Pattern Recognition. Springer, 2009.

[10] M. Jacob and M. Unser, "Design of steerable filters for feature detection using Canny-like criteria," Pattern Analysis and Machine Intelligence, IEEE Transactions on, vol. 26, no. 8, pp. 1007-1019, Aug. 2004.

[11] M. Muehlich, D. Friedrich, and T. Aach, "Design and implementation of multisteerable matched filters," Pattern Analysis and Machine Intelligence, IEEE Transactions on, vol. 34, no. 2, pp. 279-291, Feb. 2012.

[12] M. Unser and N. Chenouard, "A unifying parametric framework for 2D steerable wavelet transforms," Imaging Sciences, SIAM Journal on, vol. 6, no. 1, pp. 102-135, 2013.

[13] J. Portilla and E. P. Simoncelli, "A parametric texture model based on joint statistics of complex wavelet coefficients," Computer Vision, International Journal of, vol. 40, no. 1, pp. 49-70, Oct. 2000. 